\title{
Erratum zu: Stoffliche Nutzung von Biogasabfällen
}

PETRA SCHÖNICKE ${ }^{1}$, BIRGIT KAMM ${ }^{1}$, MIRIAM PADROCK ${ }^{2}$

${ }^{1}$ FORSCHUNGSINSTITUT BIOAKTIVE POLYMERSYSTEME E. V. (BIOPOS),TELTOW

2 BIOREFINERY.DE GMBH, TELTOW

DOI: $10.1007 / \mathrm{s} 12268-017-0860-y$

(C) Springer-Verlag 2017

Veröffentlicht: September 2017

(C) Springer-Verlag GmbH

DOI: $10.1007 / \mathrm{s} 12268-017-0842-0$

Leider ist bei der Produktion der bereits veröffentlichten Originalversion des Artikels ein Fehler in der Abbildung entstanden: „Gelöste Zucker" hat unten rechts in der Abbildung gefehlt. Anbei finden Sie die korrigierte Version.

Wir entschuldigen uns für den Fehler und für alle dadurch eventuell entstandenen Unannehmlichkeiten.

Die Online-Version des Originalartikels finden Sie unter der DOI: 10.1007/s12268-0170842-0.

Korrespondenzadresse:

Prof. Dr. Birgit Kamm

Kompetenzzentrum Holz $\mathrm{GmbH}$

Holzchemie und Biotechnologie

Science Park 2

Altenberger Straße 69

A-4040 Linz

Tel.: +43-(0)732-2468-6773

Fax: +43-(0)732-2468-6755

b.kamm@kplus-wood.at

www.kplus-wood.at

- Aufschluss von Gärresten durch Anwendung von wässrigem Ammoniak und Bioprozessen.

TS: Trockensubstanz.

Biogasgärreste

Feststoff mit ca. $25 \%$ TS

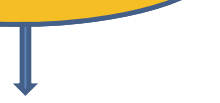

Thermaler Aufschluss

mit wässrigem Ammoniak (25\%ig)<smiles>C=[13CH]</smiles>

Extraktion

mit Wasser bei $80^{\circ} \mathrm{C}$

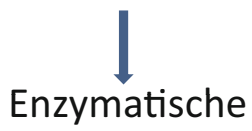

Hydrolyse

$\downarrow$

Fest/FlüssigTrennung

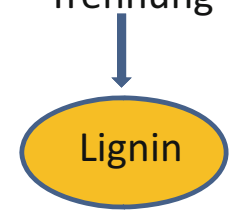

